\title{
Nachruf
}

auf

\section{HORST MENSCHING}

\author{
5. Juni 1921 - 19. Februar 2008 \\ (vorgetragen in der Plenarsitzung am 4. Februar 2011) \\ JÜRGEN HAGEDORN
}

Horst Mensching wurde am 5. Juni 1921 in Möllbergen an der Porta Westfalica geboren. Nach der Schulzeit wurde er sogleich zur Luftwaffe eingezogen, bei der er als Pilot den 2. Weltkrieg erlebte. Nach Kriegsende kehrte er nach Möllbergen zurück und konnte dann bald sein Studium mit dem Schwerpunkt Geographie in Göttingen beginnen. Dort war zu dieser Zeit das Geographische Institut, bedingt durch die frühe Wiederaufnahme des Universitätsbetriebes und relativ günstige Lebensbedingungen, ein Refugium für verschiedene angesehene deutsche Wissenschaftler. Unter ihnen war Julius Büdel, der als Privatdozent aus Berlin gekommen war und der bereits einen ausgezeichneten Ruf als Geomorphologe und Quartärforscher hatte. Er wurde Horst Menschings Doktorvater und gab ihm für ein gutes Stück seines wissenschaftlichen Weges die Richtung vor. Mensching wurde 1949 in Göttingen mit einer quartär-morphologischen Arbeit „Schotterfluren und Talauen im Niedersächsischen Bergland“ promoviert. Diese den Zeitumständen entsprechend im engen regionalen Umkreis angelegte Arbeit war dennoch von großer allgemeiner Bedeutung und stellte grundlegende Erkenntnisse bereit, die in jüngerer Zeit im Bereich der Auenforschung in der Diskussion um die klimatische und/oder die anthropogene Beeinflussung der Auensedimentation neue Aktualität erlangt haben.

Mensching war danach zunächst drei Jahre lang Wissenschaftlicher Assistent in Göttingen, ehe er 1952 seinem Lehrer Büdel nach Würzburg folgte, wo er sich noch im selben Jahr habilitierte. Seine Habilitationsschrift „Morphologische Studien im Hohen Atlas von Marokko" beruht auf intensiven Feldforschungen, die vor allem der pleistozänen Vergletscherung sowie den pleistozänen und den rezenten Periglazialerscheinungen des Gebirges wie auch den fluvialen Formen des Gebirgssaumes galten. Die klimageschichtliche Auswertung der geomorphologischen Befunde bedeu- 
tete einen wesentlichen Fortschritt in den Kenntnissen über die zeitliche und die räumliche Differenzierung des quartären Klimas am Nordrand der nordafrikanischen Trockenzone. Zugleich leistete Horst Mensching einen grundlegenden Beitrag zum Verständnis der Geomorphodynamik im semiariden Klima der Gebirgsvorländer. In zahlreichen weiteren Arbeiten, die aus Feldstudien in den Atlasländern und auf der Iberischen Halbinsel hervorgingen, hat Mensching diese Erkenntnisse vertiefen und räumlich ausweiten und damit zum Teil verallgemeinern können. Dadurch und durch weitere geomorphologische Arbeiten, die u. a. die Probleme des mediterranen Karstes und der Terra rossa und die südtunesischen Schichtstufen zum Gegenstand hatten, erwarb er sich schnell auch international einen hervorragenden Ruf als Kenner der Geomorphologie und des Quartärs im Mediterrangebiet und in dessen semiaridem Saum, insbesondere im Maghreb. Dies war die Basis für eine enge Zusammenarbeit mit französischen Forschern, insbesondere mit René Raynal.

Die Arbeiten an dieser Thematik wurden nur kurzfristig durch die von Würzburg aus durchgeführten geomorphologischen Untersuchungen in der Rhön unterbrochen, die hier deshalb genannt werden müssen, weil sie einen wichtigen Beitrag zu der gegen Ende der fünfziger Jahre sehr lebhaft geführten Diskussion über tertiäre Flächenbildung in den deutschen Mittelgebirgen darstellten. Aber Menschings hauptsächliche Forschungsregion blieb Nordafrika, auch nach seiner 1962 erfolgten Berufung auf den Geographielehrstuhl in Hannover. Hier hatte er nun die Möglichkeit, ein eigenes Forschungsteam aufzubauen und seine nordafrikanischen Forschungsaktivitäten sowohl wesentlich zu intensivieren als auch räumlich auszuweiten. Eine mehrmonatige Expedition, die die Klimazonen Nordwestafrikas von der Elfenbeinküste bis hin zum Mittelmeer durchquerte, stand 1969 am Anfang von Menschings Forschungen in der Sahelzone. Diese waren zunächst noch im Wesentlichen geomorphologisch ausgerichtet und dienten der Entwicklung des von ihm begründeten ,arid-morphodynamischen Systems" und den Fragen der Flächenbildung unter semiariden Bedingungen. Aber sie erhielten schnell eine neue Zielsetzung.

Mensching nahm seine Forschungen im Sahel 1969 auf, im selben Jahr, in dem dort die bis 1973 anhaltende große Dürrekatastrophe begann. Die Katastrophe führte zu den bis dahin deutlichsten Auswirkungen des globalen Problems der Desertifikation und weckte damit weltweit Aufmerksamkeit für diese Gefahr. Die Desertifikation sollte von nun an zum Schwerpunkt von Horst Menschings Forschungsarbeit werden. Desertifikation bedeutet nach Mensching die Ausbreitung wüstenähnlicher Verhältnisse in Gebiete hinein, in denen solche Verhältnisse zonal-klimatisch eigentlich 
nicht existieren sollten, mit ursächlicher Beteiligung des Menschen. Der Zugang zu dieser Problematik stand Mensching als Geomorphologen weit offen. Schon bei der Frage der Auenbildung in seiner Dissertation hatte er zeigen können, wie der Mensch durch seine Eingriffe in bestehende Ökosysteme zu einem geomorphologischen Faktor wurde. Es ist hinzuzufügen, dass er im weiteren Rahmen seiner Forschungen in Nordafrika von vornherein auch anthropogeographischen Aspekten Aufmerksamkeit schenkte. Dies belegen nicht zuletzt seine frühen länderkundlichen Werke über Marokko und über Tunesien - in drei Auflagen! - und dazu eine Vielzahl von Aufsätzen.

Menschings Arbeiten zur Desertifikation enthalten umfassende Analysen zu deren natürlichen und anthropogenen Ursachen und Faktoren, detaillierte Feldstudien zur Bedeutung einzelner Prozesse, die Ermittlung des Ausmaßes der Desertifikation mit Hilfe von Luft- und Satellitenbildern, die komplexe Erfassung der Prozesse und ihrer Wirkungen in enger begrenzten Nutzungsräumen mit daraus abgeleiteten Vorschlägen für Gegenmaßnahmen. Mensching hat in seinen Publikationen immer wieder die wesentlichen Ursachen der Desertifikation herausgestellt: den natürlichen Ariditätsgrad und die Niederschlagsvariabilität an den Grenzen der Trockenräume, die nichtangepasste Nutzung durch Ackerbau und Überweidung, die übermäßige Wasserentnahme auch für Bewässerungsprojekte, die Abholzung und die Brandrodung. Er hat mit der Bodenskelettierung, der Badlandbildung und der Nebka-Aufwehung frühe Zeugnisse für eine beginnende Desertifikation aufgezeigt, bei deren erstem Auftreten Gegenmaßnahmen noch leichter möglich sind. Er hat wie kaum jemand vor ihm auch die Gebirge im Hinterland arider und semiarider Gebiete und deren Naturpotential in seine Überlegungen zur Nutzung der Trockenräume unter Abwendung der Desertifikation einbezogen. Er hat schließlich sehr früh auch an historischen Beispielen die Zerstörung natürlicher Ökosysteme durch Fehlnutzung belegen können. Die Untersuchungen zur Desertifikation, die von Horst Mensching in Burkina Faso und Niger begonnen wurden, wurden von ihm nach und nach auf die gesamte Sahelzone vom Senegal bis in die Republik Sudan ausgedehnt. Damit konnte er sich in seinen Aussagen auf ungewöhnlich breite Regionalkenntnisse stützen, die im Laufe der Zeit durch Vergleichsstudien in weiteren Regionen noch ergänzt wurden.

1974 folgte Horst Mensching einem Ruf an die Universität Hamburg. Im selben Jahr wurde er in die Deutsche Akademie der Naturforscher Leopoldina und in unsere Göttinger Akademie der Wissenschaften gewählt. Darin spiegelte sich sein weiter gewachsenes Ansehen als Wissenschaftler 
wider, das auch in der 1980 erfolgten Wahl zum Chairman der Working Group „Resources Management in Drylands“ der Internationalen Geographischen Union (IGU) erkennbar wurde. Acht Jahre lang nahm er diese Aufgabe wahr und war an der Ausrichtung und der Arbeit dieser Gruppe wesentlich beteiligt. Nicht zuletzt durch vielbeachtete Symposien, z. B. in Japan und in Indien, verhalf er der Problematik der Desertifikation zu breiter Beachtung auch unter seinen Fachkollegen.

Parallel zu den wissenschaftlichen Ehrungen erfolgte die zunehmende Inanspruchnahme von Horst Mensching durch die Institutionen der nationalen und der internationalen Umweltpolitik. Als Leiter der wissenschaftlichen Beratergruppe des Bundesministeriums für wirtschaftliche Zusammenarbeit und Entwicklung (BMZ) nahm er 1977 an der UN-Conference on Desertification in Nairobi teil, war dabei Mitarbeiter am „Plan of action" und in den folgenden Jahren immer wieder Referent bei den UNEPSeminaren zur ökologischen Degradation und Desertifikation. Als Berater und Gutachter unterstütze er sowohl das BMZ und die Gesellschaft für technische Zusammenarbeit (GTZ) als auch die UNESCO bei Entwicklungsprojekten u. a. in Burkina Faso, in Jordanien, in der Republik Sudan und in Kenia. Diese praktische Umsetzung wissenschaftlicher Arbeit sah Horst Mensching als neue Herausforderung an, wobei die Projektarbeit begleitende Forschungen sicher sein wissenschaftliches Interesse wach hielten.

Schließlich ist aber auch die in dieser Zeit wichtige Rolle von Horst Mensching als unermüdlicher Werber für die Desertifikationsforschung hervorzuheben. Unter dem Eindruck der unmittelbaren Erfahrungen in den gefährdeten Regionen und der Begegnung mit den betroffenen Menschen war er überzeugt von der Pflicht der Wissenschaft zum Einsatz ihres Hilfspotentials. So setzte er sich nicht nur bei Institutionen und Organisationen für eine Verbesserung auch der vorbeugenden Forschung ein, sondern er verstand es auch, durch die Vielzahl seiner eindrucksvollen Vorträge das Interesse einer breiten Öffentlichkeit für die Probleme zu wecken. Nicht zuletzt hat er - z. B. in Seminaren der Carl-DuisbergGesellschaft und der Stiftung für Internationale Entwicklungshilfe in Khartoum (Sudan) und in Noukaschott (Mauretanien) - Aufklärungsarbeit auch an Ort und Stelle geleistet. Der Kampf gegen die Desertifikation ist nicht nur in Deutschland eng mit dem Namen und dem Schaffen von Horst Mensching verbunden.

Die Anerkennung, die der Wissenschaftler Horst Mensching fand, wird außer durch die schon genannten Ehrungen weiter durch Berufungen zum Mitglied oder Ehrenmitglied in in- und in ausländischen Akademien und geographischen Gesellschaften und durch die ihm von der Universi- 
tät Würzburg verliehene Ehrendoktorwürde dokumentiert. Andere Ehrungen waren mit der Wahrnehmung arbeitsaufwändiger Aufgaben verbunden. Die deutschen Geographen haben die internationale Erfahrung und das Ansehen von Horst Mensching genutzt, indem sie ihn für zwei Amtsperioden (1968-1972 und 1980-1984) zum Vorsitzenden ihres Nationalkomitees bei der IGU wählten und damit einen sehr tatkräftigen und effektiven Vertreter in allen internationalen Angelegenheiten des Faches fanden. Im nationalen Rahmen war er zehn Jahre lang als Fachgutachter der DFG tätig, ehe er von 1975 bis 1981 als deren Senator wirkte.

Zum Ende des Sommersemesters 1985 wurde Horst Mensching auf seinen Antrag hin emeritiert. Er hat dazu selbst ausgeführt, dass er die etwas vorzeitige Entbindung von seinen Lehrverpflichtungen in Hamburg mit der Absicht anstrebe, sich von da an verstärkt den wissenschaftlichen Aufgaben mit engem Bezug zur Praxis zu widmen, die seine Arbeitskraft ohnehin bereits überwiegend in Anspruch nahmen. Abgesehen von einem GTZ-Projekt zur Desertifikation in Patagonien, konzentrierte sich seine Forschung nunmehr vor allem auf den Sudan. Die zusammen mit Hans Poser gegründete Kommission der Akademie der Wissenschaften zu Göttingen mit dem Namen „Geomorphologische Prozesse, Prozesskombinationen und Naturkatastrophen in den Landschaftsgürteln und Höhenstufen der Erde“, kurz: „Morphodynamik“, wurde zur organisatorischen Basis seiner Forschungen. Das Forschungsprogramm der unter Menschings Leitung stehenden Arbeitsgruppe „Sudan/Sahel“ wurde von 1979 bis 1992 im Rahmen des Akademieprogramms gefördert. Es wurde von Horst Mensching vor allem mit seinen Schülern durchgeführt, darunter Doktoranden wie auch arrivierte Kollegen, und war mit ausgedehnter Geländearbeit verbunden. Die Ergebnisse sind u. a. in den sechs Bänden einer kleinen Veröffentlichungsreihe der Akademie über diese Forschungen publiziert worden. Diese Forschungen betreffen nicht nur Desertifikationsprobleme, sondern schließen auch die Analyse morphodynamischer Prozesse ein, die am Anfang von Horst Menschings Engagement in den Trockengebieten gestand hat.

Der mit dem Abschied vom Hamburger Lehrstuhl verbundenen Absicht, auf universitäre Lehrtätigkeit zu verzichten, ist Horst Mensching nur sehr eingeschränkt gefolgt. Den Kollegen am Geographischen Institut der Universität Wien gelang es nämlich, ihn von 1995 bis 2001 immer wieder zu einer einsemestrigen Gastprofessur zu verpflichten, die ganz auf seine Spezialgebiete ausgerichtet war und vor allem möglichst die Durchführung einer Exkursion in eine Trockenregion einschloss. Marokko, Patagonien, 
Namibia waren die Ziele, und noch im Herbst 2000 leitete er eine Wiener Exkursion nach Ägypten.

Horst Mensching hat sich die hervorragende gesundheitliche Konstitution, die ihn zu der Zeit noch auszuzeichnen schien und die ihm als Basis seiner Forschungen lange Jahre die Geländearbeit auch unter härtesten Bedingungen ermöglichte, nicht erhalten können. Seine letzten Jahre musste er durch Krankheit weitgehend an seine Hamburger Wohnung gefesselt und in seinen Aktivitäten völlig eingeschränkt verbringen, ehe er am 19. Februar 2008 starb. 